\title{
PROCEEDINGS
}

\section{International Symposium on Physicochemical Aspects of Polymer Surfaces held in New York City, August 24-28, 1981}

The above Symposium was held as a part of the American Chemical Society national meeting and the Division of Colloid and Surface Chemistry was the prime sponsor and it was cosponsored by Divisions of Organic Coatings and Plastics Chemistry, and Industrial and Engineering Chemistry.

The study of polymer surfaces is important from both fundamental and applied points of view, as polymers play an important role in many areas of human endeavor and all signals indicate that their usage is going to grow more and more in times to come. Wheresoever polymers are used, their surface characteristics become of paramount importance and the applications of polymers range from microelectronics to prosthetics. So the need to understand and tailor polymer surface properties is quite patent, and the availability of sophisticated surface analytical tools has been a boon in enhancing our knowledge of polymer surfaces.

In the past the topic of polymer surfaces has been discussed in various meetings but this Symposium was hailed as the most comprehensive ever held on this important topic, as the technical program contained a total of 84 papers by more than 150 authors from 21 countries representing many corners of the world. The Symposium was organized with the following objectives in mind: to bring together scientists and technologists interested in all ramifications of polymer surfaces, to review and assess the current state-ofthe-knowledge, to provide a forum for exchange and crossfertilization of ideas.

The program was divided into nine sessions: (i) Spectroscopic Analysis, (ii) Contact Angle, Wettablity and Surface Energetics, (iii) Reactions and Interactions, (iv) Tribology and Triboelectrification, (v) Adsorption and Adhesion, (vi) Crazing, Fracture and Morphology, (vii) Modifcation of Polymer Surfaces, (viii) Biomedical Aspects and Bioadhesion, and (ix) Polymer-Metal Interfaces. Even a cursory glance at the titles of the sessions should suffice to underscore that practically every aspect of polymer surfaces was covered. Even a brief description of each paper will render this Report prohibitively long so here are presented some salient comments about each session.

In the first Session a number of techniques for characterizing polymer surfaces were discussed and these included ESCA, Transmission and Reflection Spectroscopy, FT-IR, Photoacoustic FT-IR, and Auger Spectroscopy. The second session dealt with the determination of contact angles and surface free energies of polymers. The surface energy of polymers plays an important role in many areas (e.g., wetting, adhesion, printing, etc) and these were discussed. Also the surface thermodynamics of liquid polymers was discussed. The third session was devoted to reactions and interactions (thermal oxidation, photooxidation, radiation) at polymer surfaces. The papers ranged from interactions at polymer surfaces (in the form of film) to chemical reactions at the interface in polystyrene colloids.

The next session covered the tribological and triboelectrification aspects of polymers and their implications. Adsorption and adhesion of polymers constituted the theme of the next session. A number of papers discussed the adsorption at polymer surfaces, and one dealt with the acid-base character of specific interactions of polymers. The importance of polymer surface acidity and basicity was emphasized and examples were given where the acid-base concept plays an important role.

Session VI contained papers which concentrated on the crazing, fracture surface energies, and morphology of polymers. One paper specifically discussed the variation of polymer (very thin) morphology and structure through surface interactions with the substrate. The next session highlighted the various techniques for modifying polymer surfaces. The techniques discussed included: plasma (both RF and microwave), chemical (reaction with a gas, graft copolymerization), and deposition of oriented monolayers. The papers dealt with a number of different polymeric substrates and creation of differing surface chemistry.

Biomedical aspects and bioadhesion was the subject of the next session. The topics discussed included: surface energetics of polymers and bioadhesion, biomedical aspects of polymer surfaces, polymer-blood interactions, and protein adsorption at polymer surfaces. The last session was devoted to polymer-metal systems, and the papers dealt with the understanding, characterizing, and tailoring of polymer-metal interfaces. The utility of ESCA in monitoring such interfaces was amply demonstrated, and one paper discussed the use of Stoneley and leaky interface waves in characterizing metal-polymer boundaries. The last paper discussed the important issue of interfacial interactions between polymers and other materials and their effects on bond durability.

The papers discussed in this Symposium should be of interest to a broad spectrum of people with wide-ranging interests. The proceedings containing full papers will be published in two (maybe three) easily accessible volumes by the Plenum Publishing Corp. and should be available in July, 1982. It is hoped that these volumes will serve as the repository of our latest knowledge anent polymer surfaces.

Author's address:

K. L. Mittal

IBM Corp.

Hopewell Jct., N. Y. 12533

U.S.A. 